\title{
Review
}

Psychopathology

\section{Overlap between Autism Spectrum Disorder and Bipolar Affective Disorder}

\author{
Norbert Skokauskas ${ }^{\mathrm{a}, \mathrm{c}}$ Thomas Frodl ${ }^{\mathrm{b}-\mathrm{d}}$ \\ ${ }^{a}$ Centre of Child and Adolescent Mental Health and Child Protection, Department of Neuroscience, Norges \\ Teknisk-Naturvitenskapelige Universitet, Trondheim, Norway; ${ }^{\mathrm{b}}$ Department of Psychiatry, University of Regensburg, \\ Regensburg, Germany; ' Department of Psychiatry, and ${ }^{\mathrm{d}}$ Institute of Neuroscience, University of Dublin, Trinity \\ College Dublin, Dublin, Ireland
}

\section{Key Words}

Autism spectrum disorder - Bipolar affective disorder .

Co-occurrence $\cdot$ Prevalence

\begin{abstract}
Background: At present there is a substantial uncertainty regarding the extent and nature of autism spectrum disorder (ASD) and bipolar affective disorder (BPAD) co-occurrence due to disparate findings in previous studies. This paper aimed to find and review original studies on co-occurrence rates of ASD with BPAD, assess them, synthesize the findings in a systematic way, present an overview and make recommendations for future research. Methods: Systematic literature searches were performed using several databases. Selected articles had to describe an original study that provided prevalence and/or incidence analysis on ASD co-occurring together with BPAD. Results and Conclusion: A significant minority of patients (7\%) with ASD suffers from BPAD. An accurate detection of co-occurring ASD and BPAD can lead to a more targeted treatment and improve the patients' functioning and quality of life.

(c) 2015 S. Karger AG, Basel
\end{abstract}

\section{Introduction}

Autism spectrum disorder (ASD) is a lifelong neurodevelopmental disorder previously characterized as a triad of symptoms [1] and now as a dyad, comprising social communication difficulties and repetitive, stereotyped behavior [2]. A recent review of global prevalence of ASD reported a median of 62 cases per 10,000 and did not support differences in prevalence by geographic region or of a strong impact of ethnic/cultural or socioeconomic factors [3].

In the past there was a tendency to attribute almost all psychiatric and behavioral problems in persons with ASD to ASD itself [4]. Possible reasons were that the diagnosis of ASD encompasses such a severe and comprehensive label that secondary, tertiary or subsequently appearing psychiatric problems were not viewed as pivotal [5]. However, more recently an increasing number of studies have argued for accepting behaviors and symptoms that had been considered additional or associated features of ASD as potentially warranting additional diagnosis [6]. For example, Simonoff et al. [7] found in a populationbased sample of 10 - to 14 -year-old children that $71 \%$ of children with ASD met criteria for at least 1 current psychiatric disorder, $41 \%$ had 2 or more and $24 \%$ had 3 or

Prof. Norbert Skokauskas

Norges Teknisk-Naturvitenskapelige Universitet, Faculty of Medicine RKBU, PO Box 8905, MTFS NO-7491 Trondheim (Norway)

E-Mail Norbert.Skokauskas@ @ntnu.no 
more diagnoses. However, this unique ASD population study did not assess several important co-occurring psychiatric disorders, including bipolar affective disorder (BPAD). In fact, much of the literature devoted to examining the extent of co-occurring psychiatric disorders in ASD has not assessed the presence of BPAD [8].

BPAD is a familial illness characterized by recurrent episodes of mania and depression, which may have their onset in childhood. Both depressive disorders and BPAD can be specified with mixed features [2]. The current or 12-month prevalence rates of mania, hypomania and bipolar disorder in population-based studies of youth range from 0 to $0.9 \%$ in children aged $14-18$. Lifetime prevalence rates for $\mathrm{BPAD}$ among youth range from 0 to $2.1 \%$, and the lifetime prevalence rate for hypomania ranges between 0 and $0.4 \%$ [9-11]. BPAD in children is associated with significant functional impairment in several domains including increased risks for psychiatric hospitalization, antisocial behaviors, addictions and suicidal ideation $[12,13]$.

Mood disorders are one of the most common psychiatric comorbidities reported in individuals with ASD [6]. And this is despite the fact that mood changes can go easily unnoticed in many individuals with autism partly because some of them do not have sufficient language skills to express their feelings, describe changes in mood or comment on the presence of biological symptoms of depression. Even verbal individuals with ASD may be impaired in the reporting of these symptoms, given the presence of deficits in socioemotional communication. Moreover, some of the symptoms that are characteristic of ASD, e.g. social withdrawal, limited facial expression and flattened affect, are also features of depression. Typical hypomanic behaviors like overspending, gambling or excessive shopping are rarely seen in individuals with ASD. Behavior problems can manifest in other forms, i.e. verbal aggression, increased social intrusiveness, restlessness, etc. Frazier et al. [14] suggested that BPAD should be entertained as a possible co-occurring diagnosis in ASD when there is deterioration in cognition, language, behavior or activity, when there is a clear pattern of fluctuation or cyclicity in activity, behavior and interests (with 'good times' and 'bad times') and when observed behaviors indicate mood problems. A special attention should be paid at pragmatic, distracted or odd language seen in children who have language disorders as part of ASD versus the flight of ideas/thought disorder of mania [15]. Sometimes, however, bipolar symptoms are more notable and disruptive, and the intensity of the presentation of mood dysregulation masks the ASD [8].
Comorbid mood disorders in ASD were noticed early: Kanner [16] noted in his original series that at least 1 person showed a tendency to lapse into a 'momentary fit of depression', and a few children described by Asperger [17] had depressive features. The association between ASD and BPAD has only relatively recently received a larger attention despite the fact that case reports of severe periodic mood disturbances highly suggestive of BPAD have been described in children and adults with ASD for a period of time [18-23].

At present, however, there is a substantial uncertainty regarding the extent and nature of ASD and $\mathrm{BPAD}$ co-occurrence due to disparate findings in previous studies. Co-occurring conditions, especially BPAD, may more severely impair individuals with already limited cognitive functioning and social skills and may lead to increased psychological and psychosocial impairment and higher rates of hospitalization. It may also have important implications for behavioral and pharmacological treatment, given that treatment interventions for BPAD and ASD differ: while there is no established pharmacological treatment for ASD per se, there are well-known evidence-based pharmacotherapies for adult BPAD $[8,24]$. A consensus still needs to be reached regarding pharmacological treatment strategies for children with BPAD.

\section{Aims}

This paper aims to review original studies on the cooccurrence between ASD and BPAD and to provide directions for the future research to improve the diagnosis and treatment of patients with these two conditions.

\section{Methods}

PubMed, EMBASE, Science Direct, PsychINFO and Ovid Online were searched from database inception through July 2014 using the following key words: autism, ASD, Asperger's syndrome, bipolar affective disorder, BD and BPAD. Two independent unblinded reviewers (N.S. and T.F.) assessed the study eligibility by screening the titles and abstracts in a standardized manner. Then full texts were reviewed in more detail by the first author. Additional scientific papers were identified by checking the reference lists. The search was confined to English language articles. Selected articles, as a criterion for inclusion, had to describe an original study that investigated the co-occurrence of ASD and BPAD. Given the preliminary stage of research in the area, only minimal exclusion criteria were used and no limits were placed on the demographics, e.g. geographical, gender, age or size of the study populations (however, case reports were excluded). 


\section{Results}

Using the criteria described above, we identified and reviewed 20 original studies conducted in North Ameri$\mathrm{ca}$, Europe and Asia. More than a half of them [12] were published over the past 5 years reflecting the growing interest in the topic. Most studies [14] investigated children, and 3 studies focused on children with high-functioning autism (HFA) [25-27]. All studies but one [28] employed clinical samples. Most studies [18] investigated the presence of BPAD in subjects with ASD ( $\mathrm{n}=$ $6,128)$, and $431(7 \%)$ also met criteria for BPAD. Only 3 studies investigated ASD diagnosis in persons with BPAD ( $\mathrm{n}=530)$. This is not surprising as subjects with known ASD are almost always excluded from formal $\mathrm{BPAD}$ research studies. In total, 530 persons with $\mathrm{BPAD}$ were investigated, and 103 (19\%) of them met diagnostic criteria for ASD. A summary of the findings is presented in table 1.

To our knowledge, Wozniak et al. [29] were the first to report a reciprocal co-occurrence of BPAD and ASD on structured diagnostic interviews in a substantial minority of psychiatrically referred children suffering from either disorder. The 14 children with both ASD and BPAD represented $21 \%$ of the ASD group and $11 \%$ of the BPAD group. A year later Ghaziuddin et al. [30] reported on 29 males and 6 females with Asperger's syndrome (mean age 15 , range 8-51 years) who were referred to an ASD service. Of these 35 patients, 23 (66\%) had an additional psychiatric disorder and 1 patient had BPAD.

A study by Stahlberg et al. [31] described the prevalence and patterns of co-occurring bipolar and psychotic disorders in 241 consecutively referred adult patients with attention-deficit/hyperactivity disorder and/or ASD. Nine (7.0\%) of the 129 subjects with ASD had bipolar disorder with psychotic features [31]. Towbin et al. [32] screened 93 patients aged $8-18$ in a mood disorder clinic in Maryland, USA, using the Social Communication Questionnaire (SCQ), Children's Communication Checklist (CCC), the Social Reciprocity Scale (SRS) and a published preliminary report [32]. Few years later this group gathered data on more patients (259) and published the final study with 352 participants of whom 62 had BPAD. The SRS-based cutoff for ASD was met by 33 (56.9\%) patients with BPAD, the SRC-based one by 4 (8.3\%) and the CCC-based one by 23 (53.5\%) [33].

Leyfer et al. [34] assessed 109 children with ASD, and 3 of them had BPAD (BPAD 1 - two and BPAD 2 - one). The relative advantage of the approach used here was the attempt to standardize diagnoses of comorbidity using

Co-Occurrence of ASD and BPAD the Autism Comorbidity Interview (ACI), a modified version of the Kiddie Schedule for Affective Disorders and Schizophrenia [34]. According to Leyfer et al., the ACI provides a more accurate measure of the behavioral manifestation of psychiatric comorbidity by gathering information on symptoms that cluster together in time, are significantly impairing, and, when acute, represent a change from the child's baseline emotional state, behavior and functioning [34, 35]. Mazefsky et al. [36] administered the ACI to 35 children with ASD and compared the findings to their history of prior diagnoses to help clarify the level of concordance. Approximately $60 \%$ of prior psychiatric diagnoses were not supported on the ACI; the lowest diagnostic concordance was for prior BPAD and obsessive-compulsive disorder diagnoses. In fact, none of $14.3 \%$ [5] BPAD community diagnoses was supported. Interestingly, medication use was especially pronounced among participants who had been previously diagnosed with BPAD (mean number attempted $=7.0$ ) [36].

Bradley and Bolton [28] conducted the first study to document an increased prevalence of psychiatric illness associated with ASD in an epidemiological sample of individuals with learning disabilities. Two $(5.5 \%)$ in the ASD group $(\mathrm{n}=36)$ were diagnosed with BPAD compared with none in the control group (teenagers with other learning disabilities). In Japan Munesue et al. [26] reported a higher rate of BPAD in patients with high-functioning ASD. Munesue et al. examined for mood disorders 44 consecutive outpatients with high-functioning ASD at a university hospital using DSM-IV criteria. Sixteen patients (36.4\%) were diagnosed with mood disorder. More patients were diagnosed with BPAD [13] than depression [4]. Two patients were diagnosed with BPAD 1, 6 patients with BPAD 2 and 4 with BPAD not otherwise specified. Of these 16 patients identified also as having mood disorders, 12 had Asperger's disorder as primary diagnosis, 4 pervasive developmental disorder not otherwise specified and none autistic disorder as per DSM-IV [26].

In 2004 Bryson et al. [37] examined 1 year of data from the database maintained by 26 community mental health centers in Kansas State, USA: 586 children with ASD were identified, and 48 of them (8.2\%) had a diagnosis of BPAD. Rosenberg et al. [38] went a step further and examined a national US online registry to assess variation in cumulative prevalence of community diagnosis of psychiatric comorbidity in 4,343 children with ASD. They reported lower rates of co-occurrence between ASD and $\mathrm{BPAD}$ in comparison to the study of Bryson et al. [37]. In fact, in the study of Rosenberg et al. [38], BPAD was one 
Table 1. Co-occurrence of ASD and BPAD

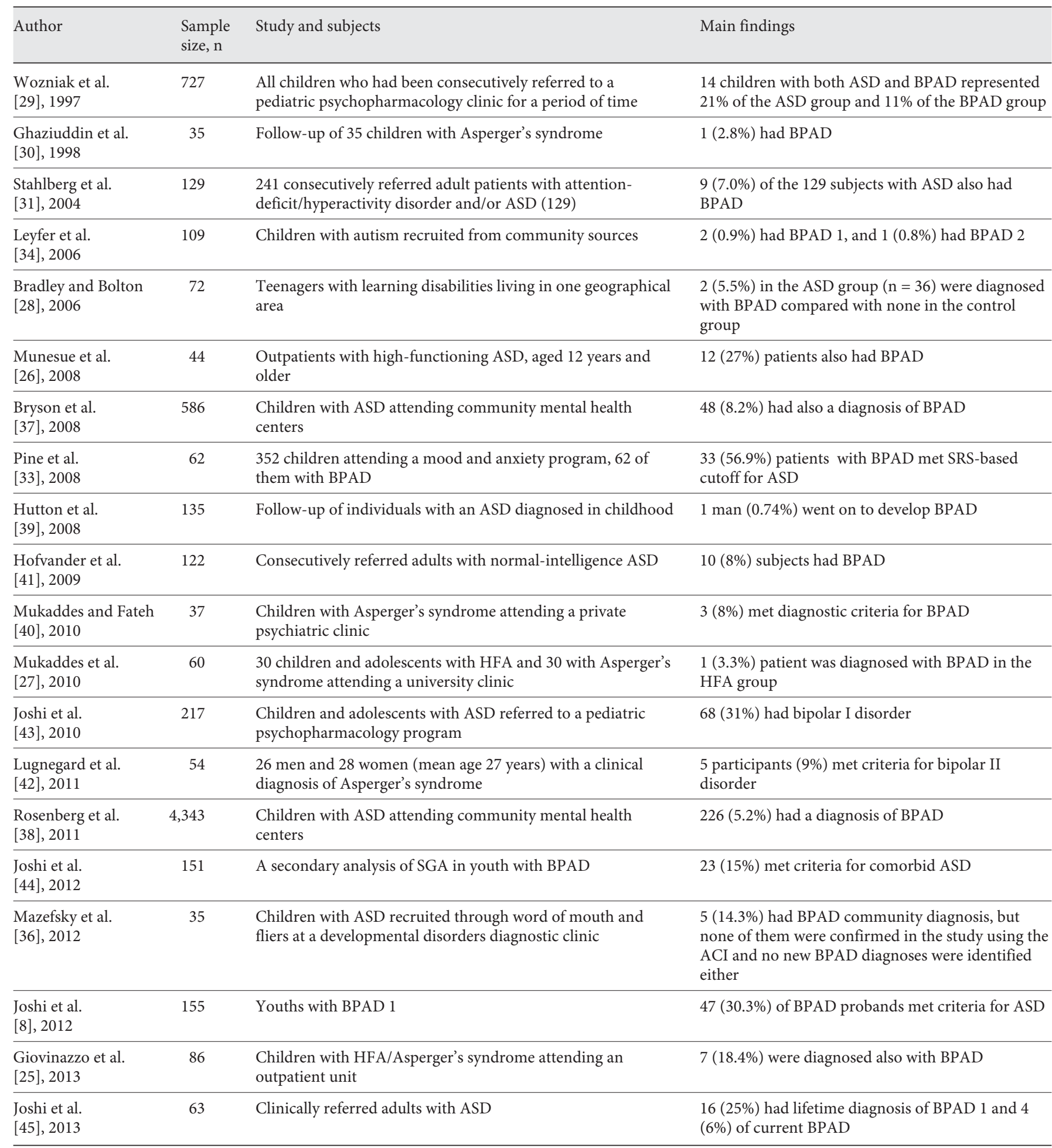

SRS = Social Reciprocity Scale; SGA = second-generation antipsychotics; ACI = Autism Comorbidity Interview. 
of the least common co-occurring diagnoses (5.2\%), with attention-deficit/hyperactivity disorder or attention deficit disorder (38.1\%) being the most common [37].

In the UK, Hutton et al. [39] performed a follow-up study of 135 individuals aged at least 21 years with an ASD diagnosed in childhood. They reported that only 1 man $(0.74 \%)$ went on to develop BPAD.

Mukkades et al. [27, 40] have recently published 2 papers on co-occurring conditions in ASD. In the first study they aimed to provide clinical data regarding co-occurring psychiatric disorders in children diagnosed with Asperger's disorder. Mukkades et al. used the Schedule for Affective Disorders and Schizophrenia for School Age Children Present and Lifetime Version (K-SADS-PL-T) to assess 32 boys and 5 girls with $\mathrm{AD}$ attending a private psychiatric clinic [40]. 94\% of participants had at least 1 additional psychiatric disorder, and $3(8 \%)$ met diagnostic criteria for BPAD. In the second study, Mukkades et al. assessed 30 children and adolescents with HFA and 30 with ASD for co-occurring psychiatric disorders using the K-SADS-PL-T. These patients were recruited from a public clinic. The rate of co-occurring psychiatric disorders was very high in both groups (93.3\% in HFA and $100 \%$ in AS). However, only 1 (3.3\%) patient was diagnosed with BPAD in the HFA group and no patients were diagnosed with BPAD in the ASD group [27]. A recent Italian study also investigated the co-occurrence of psychiatric disorders in 86 children with HFA and AS attending a university clinic. 38 patients out of $86(44.2 \%)$ presented with 1 or more additional psychiatric conditions and $7(18.4 \%)$ were diagnosed with BPAD [25]. Hofvander et al. [41] assessed 122 consecutively referred adults with normal-intelligence ASD. This study group consists of subjects pooled from 2 studies with similar protocols, all seen on an outpatient basis. Criteria for a bipolar disorder were met by 10 subjects (8\%), 5 of whom had BPAD 1 subtype and 2 BPAD 2, while 3 were coded as unknown subtypes. No subject with Asperger's disorder met criteria for BPAD. Only 3 patients $(2 \%)$ had ever been treated with a mood stabilizer [41].

Lugnegard et al. investigated the psychiatric co-occurrence in young adults with Asperger's syndrome. Participants were 26 men and 28 women (mean age 27 years) with a clinical diagnosis of Asperger's syndrome. Psychiatric comorbidity was assessed by the Structured Clinical Interview for DSM-IV Axis I Disorders [42]. ASD was confirmed using the Diagnostic Interview for Social and Communication Disorders. Five participants (9\%) met criteria for BPAD 2, whereas none met criteria for BPAD 1. Of these 5, only 1 had an already previously established

Co-Occurrence of ASD and BPAD clinical diagnosis of BPAD and a history of mood-stabilizing treatment.

Recently, Joshi et al. [43] have conducted several studies which among other things investigated the co-occurrence of BPAD and ASD. In 2010, Joshi et al. compared youth-satisfying diagnostic criteria for ASD and age- and sex-matched youth without ASD referred to the same clinical program. $9.3 \%(217 / 2,323)$ of the referred youth (age range: 3-17 years) met DSM-III-R criteria for ASD. BPAD 1 disorder was diagnosed in $68(31 \%)$ subjects with ASD and 65 (30\%) controls [43]. A year later Joshi et al. performed a secondary analysis of identically designed 8-week open-label trials of second-generation antipsychotic monotherapy (risperidone, olanzapine, quetiapine, ziprasidone or aripiprazole) in youth with BPAD. Out of 151 BPAD subjects, $15 \%(n=23)$ met criteria for comorbid ASD. No difference was observed in the rate of antimanic response or tolerability to second-generation antipsychotic monotherapy in the presence of ASD comorbidity [44]. In the same year, Joshi et al. conducted a systematic assessment of phenotypic and familial correlates of BPAD 1 in youth with and without ASD to investigate whether presentation of mania in youth with comorbid ASD truly represents BPAD. Findings revealed that the phenotypic features of BPAD 1 disorder were largely similar in youth with BPAD 1 disorder irrespective of comorbidity with ASD [8]. Last year, Joshi et al. examined 63 clinically referred adults with ASD. All ASD participants, derived from the ASD clinic, received a neuropsychological assessment, a structured diagnostic interview and a psychiatric interview. A primary caretaker (if available) also completed a structured diagnostic interview. 16 (25\%) had a lifetime diagnosis of BPAD 1 and 4 (6\%) a current BPAD 1 diagnosis. In comparison, 8 (13\%) in the control group met criteria for lifetime diagnosis of BPAD and 13 (5\%) current BPAD 1 diagnosis [45].

\section{Discussion}

Until relatively recently, BPAD and ASD co-occurrence has received relatively little attention in the research literature. This, to our knowledge, is the first paper to review published studies on the co-occurrence of ASD and BPAD.

Our review has identified a very broad diapason of BPAD and ASD co-occurrence ranging from 0 to $56.9 \%$. Given the variable approaches used, this is not surprising. Such a wide range may also suggest that some studies reviewed reported 'artificial' co-occurrence. Sources of ar- 
tifact in the detection of co-occurrence between psychiatric disorders have been well described previously and include referral, screening and surveillance factors, the concepts of disorders as categories or dimensions, overlapping diagnostic criteria, artificial subdivisions of syndromes, one disorder representing an early manifestation of the other, and one disorder being part of the other [46]. In brief, there are 2 main types of an artificial co-occurrence: predisposed by study design and diagnostic concepts. Both BPAD and ASD in particular are quite heterogeneous, which further contributes to nosological complexities. Indeed, one of the major difficulties in studying co-occurrence is the lack of validated diagnostic criteria [47]. However, validity of diagnostic criteria and previously used classification systems and concepts (i.e. DSMIV) are beyond the scope of this paper. Hence, below we will focus on limitations of the studies reviewed which could have contributed to reporting artificial co-occurrence.

The studies reporting very high $[33,34]$ or no co-occurrence [36] had several methodological shortcomings. First, the study by Pine et al. [33], which reported the highest rates of ASD, was based only on screens (SCQ, CCC and SRS). The Autism Diagnostic Observation Schedule and the Autism Diagnostic Interview Revised which are considered to be the 'gold standard' in diagnostic evaluations for ASD were not employed [33]. Although developers of the SCQ, CCC and SRS consider them as ASD screens for various populations, questions remain as to the degree to which they measure distinct constructs and central features of ASD. Moreover in the study of Pine et al., the sample consisted only of youths receiving treatment and eligible for participation in biological studies. Such samples are not representative of children in the community. And finally this study performed assessment on ASD symptom scales only at one point in time. This is, however, a common constraint for many of the reviewed studies: they did not follow up individuals diagnosed in childhood into adulthood and thus were not in a position to study the pattern of disorder and co-occurring conditions in adult life. Interestingly, the two studies $[30,39]$ which followed up children with ASD reported very low rates of co-occurrence (2.8 and $0.74 \%)$. However, it has to be noted that the sample employed by Ghaziuddin et al. [30] was the second smallest $(\mathrm{n}=35)$ in this review. Indeed, a small and very selective clinical sample size with no power considerations is a common trait of many studies reviewed.

The study by Mazefsky et al. [36], which used the ACI and reported no co-occurrence between ASD and BPAD, had also some methodological limitations. $60 \%$ of prior psychiatric diagnoses were not supported on the ACI, and none of $14.3 \%$ [5] BPAD community diagnoses was supported. This raises at least two questions, first how community diagnoses were established and reported and how reliable the ACI is. Prior psychiatric diagnoses in this study were determined only on parent/guardian report. The lack of concordance may reflect a diminished sensitivity of the ACI. It is hard to compare how the ACI performed in other studies as nobody else but its creators [34] and Mazefsky et al. [36] have used it. The study also employed a very selective clinical sample of pre-adolescents and adolescents with HFA, with the majority not receiving their ASD diagnosis until late childhood or adolescence. Still the other studies which investigated HFA reported much higher co-occurrence rates [25-27].

It has to be emphasized, however, that several studies noted the clinical presentation of BPAD and ASD in the co-occurrence state to be strikingly analogous to their typical presentation, suggesting a bona fide co-occurrence of these disorders $[26,29,43]$. Notably, studies with the largest ASD samples - 241 [31], 586 [37] and 4,343 [38] - reported quite similar co-occurrence rates of $7,8.2$ and $5.2 \%$. Indeed, $7 \%$ was the mean prevalence rate of BPAD in ASD based on all studies reviewed. This is much higher in comparison to the general population and it would also imply that $7 \%$ of subjects with ASD might also need treatment for BPAD to improve their wellbeing and quality of life.

The real co-occurrence (vs. 'artificial') can be predisposed by at least several factors: shared environmental or biological risk factors; overlapping risk factors; comorbid patterns constituting a meaningful syndrome, and one disorder creating an increased risk for the other [46]. Indeed, the results of family studies suggested that a significant relationship may exist between ASD and affective disorders. DeLong and Nohria [48] reported that in 20 individuals with ASD, 14 had a positive family history of major affective disorder; in the 14 families, 14 (11.4\%) of 123 relatives had bipolar disorder and an additional 19 (15.4\%) had a history of unipolar depression. Last year the Genomics Consortium described its analysis of genome-wide single nucleotide polymorphism data for 33,332 cases and 27,888 controls distributed among the 5 major psychiatric disorders in the Genomics Consortium (major depressive disorder, BPAD, schizophrenia, ASD and attention-deficit/hyperactivity disorder). Aggregate polygenic risk scores for a broad set of common variants showed cross-disorder effects for all the adult-onset disorders (bipolar and major depressive disorder, and 
schizophrenia) and nominally between autism spectrum disorders and both bipolar disorder and schizophrenia [49].

More recently, neuroimaging, inflammation, maternal infection and other studies questioning a possibility of shared biological factors between BPAD and ASD have started to emerge.

Perlman et al. [50] reported hypoactivation, isolated to the fusiform gyrus, when viewing animated, emerging facial expressions of happiness, sadness, fearfulness and especially anger in pediatric BPAD patients relative to matched clinical and healthy control groups. This region is widely noted as a region involved not only in face processing but in key components of social communication, such as eye contact, facial identity recognition and mouth movement. Although this region has rarely been studied directly in pediatric BPAD, inferences can be drawn from widely replicated results of hypoactivation and social impairment in the ASD literature [50]. A recent systematic review and meta-analysis reported significantly higher concentrations of interleukins $1 \beta, 6,8$, interferon- $\gamma$, eotaxin and monocyte chemotactic protein-1 in the participants with ASD compared with healthy controls [51]. Birmaher et al. [52] reported in youth with BPAD an asso- ciation of high-sensitivity C-reactive protein but not interleukin-6 with manic symptom severity, although this association was reduced to a trend when obesity was included in multivariate analysis. The plausibility of a pathophysiological relationship between BPAD and inflammation is further reinforced by evidence that a proinflammatory gene expression signature preceded the first mood episode in previously healthy offspring of adults with BPAD [53]. Parboosing et al. [54] reported nearly a 4-fold increase in the risk of BPAD exposure to maternal influenza at any time during pregnancy. Atladottir et al. [55] reported a statistically significant association between maternal viral infection during the first trimester and ASD in the child. However, no association was found between any maternal infection and diagnosis of ASD in the child when looking at the total period of pregnancy.

In conclusion, a significant minority of patients suffer from both ASD and BPAD. An accurate detection of cooccurring ASD and BPAD can lead to a more targeted treatment and improve patient functioning and quality of life. The complexity of BPAD and ASD co-occurrence highlighted by this paper requires larger and better designed studies with appropriate assessment tools and a longitudinal approach.

\section{References}

1 American Psychiatric Association: Diagnostic and Statistical Manual of Mental Disorders, ed 4. Washington, American Psychiatric Association, 2000.

2 American Psychiatric Association: Diagnostic and Statistical Manual of Mental Disorders, ed 5. Washington, American Psychiatric Association, 2013.

3 Elsabbagh M, Divan G, Koh YJ, Kim YS, Kauchali S, Marcin C, et al: Global prevalence of autism and other pervasive developmental disorders. Autism Res 2012;5:160-179.

4 Lainhart JE: Psychiatric problems in individuals with autism, their parents and siblings. Int Rev Psychiatry 1999;11:278-298.

5 Bolte S, Bosch G: The long-term outcome in two females with autism spectrum disorder. Psychopathology 2005;38:151-154.

6 Skokauskas N, Gallagher L: Psychosis, affective disorders and anxiety in autistic spectrum disorder: prevalence and nosological considerations. Psychopathology 2010;43:8-16.

7 Simonoff E, Pickles A, Charman T, Chandler S, Loucas T, Baird G: Psychiatric disorders in children with autism spectrum disorders: prevalence, comorbidity, and associated factors in a population-derived sample. J Am Acad Child Adolesc Psychiatry 2008;47:921-929.
8 Joshi G, Biederman J, Petty C, Goldin RL, Wozniak J: Examining the comorbidity of bipolar disorder and autism spectrum disorders: a large controlled analysis of phenotypic and familial correlates in youth with bipolar disorder. Eur Psychiatry 2012;27:1.

9 Costello EJ, Mustillo S, Keller G, Angold A: Prevalence of psychiatric disorders in childhood and adolescence; in Levin BL, Petrila J, Hennessy KD (eds): Mental Health Services: A Public Health Perspective, ed 2. Oxford, Oxford University Press, 2004, pp 111-128.

10 Lewinsohn PM, Rohde P, Seeley JR, Fischer SA: Age-cohort changes in the lifetime occurrence of depression and other mental disorders. J Abnorm Psychol 1993;102:110-120.

11 Merikangas KR, Nakamura EF, Kessler RC: Epidemiology of mental disorders in children and adolescents. Dial Clin Neurosci 2009;11: 7-20.

12 Wozniak J, Biederman J, Kiely K, Ablon JS, Faraone SV, Mundy E, et al: Mania-like symptoms suggestive of childhood-onset bipolar disorder in clinically referred children. J Am Acad Child Adolesc Psychiatry 1995;34:867876.
13 Birmaher B, Axelson D, Strober M, Gill MK, Valeri S, Chiappetta L, et al: Clinical course of children and adolescents with bipolar spectrum disorders. Arch General Psychiatry 2006;63:175-183.

14 Frazier JA, Doyle R, Chiu S, Coyle JT: Treating a child with Asperger's disorder and comorbid bipolar disorder. Am J Psychiatry 2002;159:13-21.

15 Carlson GA: Differential diagnosis of bipolar disorder in children and adolescents. World Psychiatry 2012;11:146-152.

16 Kanner L: Autistic disturbance of affective contact. Nerv Child 1943;2:217-250.

17 Asperger H: Die autistischen Psychopathen im Kindesalter. Arch Psychiatr Nervenkrankh 1944;117:76-136.

18 Komoto J, Usui S, Hirata J: Infantile autism and affective disorder. J Autism Dev Disord 1984;14:81-84.

19 Steingard R, Biederman J: Lithium responsive manic-like symptoms in two individuals with autism and mental retardation. J Am Acad Child Adolesc Psychiatry 1987;26:932-935.

20 Wing L: Asperger's syndrome: a clinical account. Psychol Med 1981;11:115-129.

21 Gillberg C: Asperger's syndrome and recurrent psychosis - a case study. J Autism Dev Disord 1985;15:389-397. 
22 Lainhart JE, Folstein SE: Affective disorders in people with autism: a review of published cases. J Autism Dev Disord 1994;24:587601.

23 Raja M, Azzoni A: Comorbidity of Asperger's syndrome and bipolar disorder. Clin Pract Epidemiol Mental Health 2008;4:26.

24 Weissman A, Bates ME: Increased clinical and neurocognitive impairment in children with autism spectrum disorders and comorbid bipolar disorder. Res Autism Spectrum Disord 2010;4:670-680.

25 Giovinazzo S, Marciano S, Giana G, Curatolo P, Porfirio M-C: Clinical and therapeutic implications of psychiatric comorbidity in high functioning autism/Asperger syndrome: an Italian study. Open J Psychiatry 2013;3:329334.

26 Munesue T, Ono Y, Mutoh K, Shimoda K, Nakatani H, Kikuchi M: High prevalence of bipolar disorder comorbidity in adolescents and young adults with high-functioning autism spectrum disorder: a preliminary study of 44 outpatients. J Affect Disord 2008;111: 170-175.

27 Mukaddes NM, Herguner S, Tanidir C: Psychiatric disorders in individuals with highfunctioning autism and Asperger's disorder: similarities and differences. World J Biol Psychiatry 2010;11:964-971.

28 Bradley E, Bolton P: Episodic psychiatric disorders in teenagers with learning disabilities with and without autism. Br J Psychiatry 2006;189:361-366.

29 Wozniak J, Biederman J, Faraone SV, Frazier J, Kim J, Millstein R, et al: Mania in children with pervasive developmental disorder revisited. J Am Acad Child Adolesc Psychiatry 1997;36:1552-1559, discussion 1559-1560.

30 Ghaziuddin M, Weidmer-Mikhail E, Ghaziuddin N: Comorbidity of Asperger syndrome: a preliminary report. J Intellect Disabil Res 1998;42:279-283.

31 Stahlberg O, Soderstrom H, Rastam M, Gillberg C: Bipolar disorder, schizophrenia, and other psychotic disorders in adults with childhood onset $\mathrm{AD} / \mathrm{HD}$ and/or autism spectrum disorders. J Neural Transm 2004;111:891902.

32 Towbin KE, Pradella A, Gorrindo T, Pine DS, Leibenluft E: Autism spectrum traits in children with mood and anxiety disorders. J Child Adolesc Psychopharmacol 2005; 15:452-464.

33 Pine DS, Guyer AE, Goldwin M, Towbin KA, Leibenluft E: Autism spectrum disorder scale scores in pediatric mood and anxiety disorders. J Am Acad Child Adolesc Psychiatry 2008;47:652-661.
34 Leyfer OT, Folstein SE, Bacalman S, Davis NO, Dinh E, Morgan J, et al: Comorbid psychiatric disorders in children with autism: interview development and rates of disorders. J Autism Dev Disord 2006;36:849-861.

35 Leyfer OT, Tager-Flusberg H, Dowd M, Tomblin JB, Folstein SE: Overlap between autism and specific language impairment: comparison of Autism Diagnostic Interview and Autism Diagnostic Observation Schedule scores. Autism Res 2008;1:284-296.

36 Mazefsky CA, Oswald DP, Day TN, Eack SM, Minshew NJ, Lainhart JE: ASD, a psychiatric disorder, or both? Psychiatric diagnoses in adolescents with high-functioning ASD. J Clin Child Adolesc Psychol 2012;41:516-523.

37 Bryson SA, Corrigan SK, McDonald TP, Holmes C: Characteristics of children with autism spectrum disorders who received services through community mental health centers. Autism 2008; $12: 65-82$

38 Rosenberg RE, Kaufmann WE, Law JK, Law PA: Parent report of community psychiatric comorbid diagnoses in autism spectrum disorders. Autism Res Treat 2011;2011:405849.

39 Hutton J, Goode S, Murphy M, Le Couteur A, Rutter M: New-onset psychiatric disorders in individuals with autism. Autism 2008;12: 373-390.

40 Mukaddes NM, Fateh R: High rates of psychiatric co-morbidity in individuals with Asperger's disorder. World J Biol Psychiatry 2010;11:486-492.

41 Hofvander B, Delorme R, Chaste P, Nyden A, Wentz E, Stahlberg O, et al: Psychiatric and psychosocial problems in adults with normalintelligence autism spectrum disorders. BMC Psychiatry 2009;9:35.

42 Lugnegard T, Hallerback MU, Gillberg C: Psychiatric comorbidity in young adults with a clinical diagnosis of Asperger syndrome. Res Dev Disabil 2011;32:1910-1917.

43 Joshi G, Petty C, Wozniak J, Henin A, Fried $\mathrm{R}$, Galdo M, et al: The heavy burden of psychiatric comorbidity in youth with autism spectrum disorders: a large comparative study of a psychiatrically referred population. J Autism Dev Disord 2010;40:1361-1370.

44 Joshi G, Biederman J, Wozniak J, Doyle R, Hammerness P, Galdo M, et al: Response to second generation antipsychotics in youth with comorbid bipolar disorder and autism spectrum disorder. CNS Neurosci Ther 2012; 18:28-33.
45 Joshi G, Wozniak J, Petty C, Martelon MK, Fried R, Bolfek A, et al: Psychiatric comorbidity and functioning in a clinically referred population of adults with autism spectrum disorders: a comparative study. J Autism Dev Disord 2013;43:1314-1325.

46 Caron C, Rutter M: Comorbidity in child psychopathology: concepts, issues and research strategies. J Child Psychol Psychiatry Allied Disc 1991;32:1063-1080.

47 Bukstein OG, Brent DA, Kaminer Y: Comorbidity of substance abuse and other psychiatric disorders in adolescents. Am J Psychiatry 1989;146:1131-1141.

48 DeLong R, Nohria C: Psychiatric family history and neurological disease in autistic spectrum disorders. Dev Med Child Neurol 1994; 36:441-448.

49 Cross-Disorder Group of the Psychiatric Genomics: Identification of risk loci with shared effects on five major psychiatric disorders: a genome-wide analysis. Lancet 2013;381: 1371-1379.

50 Perlman SB, Fournier JC, Bebko G, Bertocci MA, Hinze AK, Bonar L, et al: Emotional face processing in pediatric bipolar disorder: evidence for functional impairments in the fusiform gyrus. J Am Acad Child Adolesc Psychiatry 2013;52:1314-1325.e3.

51 Masi A, Quintana DS, Glozier N, Lloyd AR, Hickie IB, Guastella AJ: Cytokine aberrations in autism spectrum disorder: a systematic review and meta-analysis. Mol Psychiatry 2015; 20:440-446.

52 Birmaher B, Axelson D, Goldstein B, Strober M, Gill MK, Hunt J, et al: Four-year longitudinal course of children and adolescents with bipolar spectrum disorders: the Course and Outcome of Bipolar Youth (COBY) study. Am J Psychiatry 2009; 166:795-804.

53 Padmos RC, Hillegers MH, Knijff EM, Vonk R, Bouvy A, Staal FJ, et al: A discriminating messenger RNA signature for bipolar disorder formed by an aberrant expression of inflammatory genes in monocytes. Arch Gen Psychiatry 2008;65:395-407.

54 Parboosing R, Bao Y, Shen L, Schaefer CA, Brown AS: Gestational influenza and bipolar disorder in adult offspring. JAMA Psychiatry 2013;70:677-685.

55 Atladottir HO, Thorsen P, Ostergaard L, Schendel DE, Lemcke S, Abdallah M, et al: Maternal infection requiring hospitalization during pregnancy and autism spectrum disorders. J Autism Dev Disord 2010;40:14231430. 